\title{
A Rare Case of Intracerebral Pneumocephalus Caused by Preexisting Multiple Bone Defects and Encephalocele after Resection of Meningioma
}

\author{
Dan OZAKI, ${ }^{1}$ Toshiaki AKASHI, ${ }^{2}$ Takahiro MORITA, ${ }^{1}$ Shinjitsu NisHIMURA, ${ }^{3}$ \\ Masayuki KANAMORI, ${ }^{1}$ and Teiji TOMINAGA ${ }^{1}$ \\ ${ }^{1}$ Department of Neurosurgery, Tohoku University Graduate School of Medicine, Sendai, \\ Miyagi, Japan \\ ${ }^{2}$ Department of Diagnostic Radiology, Tohoku University Graduate School of Medicine, \\ Sendai, Miyagi, Japan \\ ${ }^{3}$ Department of Neurosurgery, Minami Tohoku Hospital, Iwanuma, Miyagi, Japan
}

\begin{abstract}
Pneumocephalus is generally secondary to direct damage to the skull base. Spontaneous intracerebral pneumatocele without head injury was extremely rare, but previously reported as a serious complication of shunt procedures. We describe a 40-year-old man with intracerebral pneumocephalus who previously underwent craniotomy for large frontal convexity meningioma and lumbo-peritoneal shunting. He presented with gait disturbance 14 months after tumor resection. Computed tomography and magnetic resonance imaging showed intracerebral pneumocephalus in the right temporal lobe, which continued into the mastoid air cells through a bone defect of the right petrous bone. We performed urgent right temporal craniotomy to reduce the mass effect and to repair the fistula. Intraoperatively, bone defects were identified at the roof petrous bone, into which the encephalocele had penetrated. The herniated cerebral parenchyma was removed, and the pneumocephalus opened. The dura was closed with sutures and covered with fascia. To elucidate the underlying mechanism for the development of intracranial pneumocephalus, the previous images obtained before or immediately after resection of meningioma were reviewed. We founded that multiple preexisting bone defects and encephaloceles, one of which was considered to be the cause of the intracerebral pneumocephalus. This case demonstrates that intracerebral pneumocephalus can be caused by preexisting bone defect and encephalocele, and this finding may be useful for prediction of pneumocephalus after shunt procedures.
\end{abstract}

Keywords: pneumocephalus, meningioma, lumbo-peritoneal shunting, encephalocele

\section{Introduction}

Pneumocephalus is generally a result of skull base fracture, bone defect caused by head trauma, surgical procedure, or tumor invasion or infection around the frontal base or petrous bone. ${ }^{1)}$ However, pneumocephalus may develop spontaneously without direct injury to the skull base. ${ }^{1-10)}$ One of the important pathogeneses for spontaneous pneumocephalus is an imbalance between the intracranial pressure (ICP) and extracranial pressure caused by decreased ICP

Received March 11, 2020; Accepted May 1, 2020

Copyright $\odot 2021$ by The Japan Neurosurgical Society This work is licensed under a Creative Commons AttributionNonCommercial-NoDerivatives International License. resulting from ventriculo-peritoneal (VP) shunting ${ }^{2-4,7-9,11,12)}$ and intracranial hypotension, ${ }^{5)}$ or increased pressure in the middle ear, mastoid air cells, or paranasal cavity resulting from changes in altitude ${ }^{6)}$ or bilevel positive airway pressure. ${ }^{13)}$

Pneumocephalus is rare, but is a serious complication of shunt procedures, so prediction and prevention are highly desirable. Most of the cases presenting pneumocephalus after shunting had chronic elevated ICP ${ }^{2,4,7-9,11,12)}$ before shunting. Previous intraoperative findings have revealed destruction of the dura and/or encephalocele into bone defect as causes of pneumocephalus. Such findings seem to be useful for prediction, but no case has shown these preexisting findings before shunt procedure. 


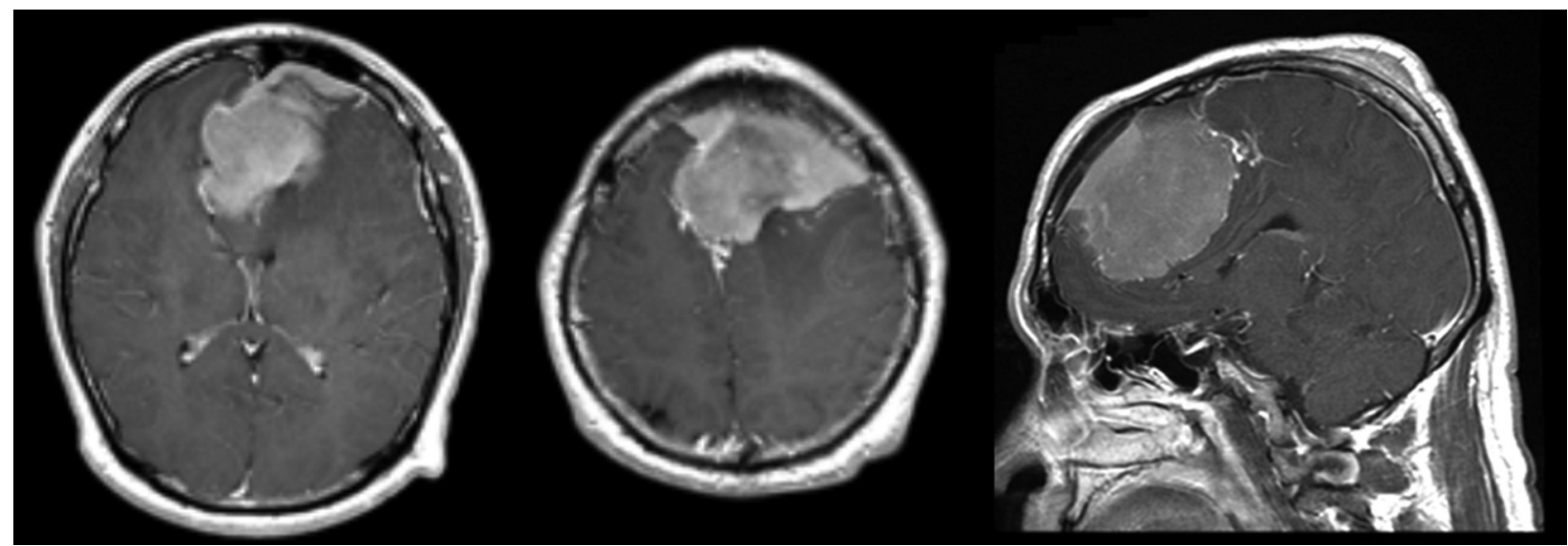

Fig. 1 Axial (left and middle panels) and sagittal (right panel) gadolinium-enhanced T1-weighted MR images demonstrating left frontal convexity meningioma. MR: magnetic resonance.

We describe a case of pneumocephalus in the right temporal lobe, which occurred 14 months after removal of left frontal convexity meningioma followed by lumbo-peritoneal (LP) shunting, which demonstrated useful findings for the prediction of intracerebral pneumocephalus after the shunt procedure.

\section{Case Report}

A 39-year-old man presented with headache and left frontal convexity meningioma was identified (Fig. 1). He underwent left frontal craniectomy, resection of the tumor, and cranioplasty with titanium mesh due to skull invasion by the meningioma. He underwent LP shunting for intractable subcutaneous cerebrospinal fluid collection in the frontal area 6 months after tumor resection. He subsequently presented with gait disturbance and was hospitalized at 14 months after tumor resection with left hemiparesis, left hemi-spatial neglect, and left homonymous hemianopsia.

Computed tomography (CT) showed intracerebral pneumocephalus in the right temporal lobe (Fig. 2A). The intracerebral pneumocephalus continued into the mastoid air cells through a bone defect of the right petrous bone, which was independent of the craniectomy (Fig. 2B). Physical, laboratory, and magnetic resonance (MR) imaging findings detected no indication of infection. We performed urgent right temporal craniotomy to reduce the mass effect of tension pneumocephalus and to repair the fistula. Bone defects were identified at the roof of petrous bone, into which the encephalocele had penetrated (Fig. 2C). The surrounding petrous bone was drilled, and the encephalocele was exposed completely (Fig. 2C). The herniated cerebral parenchyma was removed, and the pneumocephalus opened. The dura was closed with sutures and covered with fascia of the temporal muscle. Postoperative course was uneventful, and he was discharged with incomplete left homonymous hemianopsia.

We reviewed the previous images to elucidate the underlying mechanism for the development of intracranial pneumocephalus. CT before resection and three-dimensional CUBE T2-weighted MR imaging 2 days after tumor resection found the bone defect in the roof of the mastoid air cells and encephaloceles (Fig. 3A). Combined with the finding of intracerebral pneumocephalus (Figs. 2B and 3B), this lesion was considered to be the cause of the intracerebral pneumocephalus. In addition, other multiple skull defects were identified at the bilateral temporal bones before resection of meningioma (Fig. 3) and encephalocele in the bilateral calvaria and left petrous bone 2 days after resection of the meningioma (Fig. 3D).

\section{Discussion}

In this report, we presented the case of intracerebral pneumocephalus caused by preexisting multiple bone defects and encephalocele after resection of meningioma. In previous report, nine cases including our case have been reported as tumor related intracerebral pneumocephalus (Table 1).,2,8,8,14,15) Tumor location was supratentorial in four and infratentorial in five cases. Histological diagnosis was meningioma in three, neurinoma in three, astrocytoma in one, cavernous hemangioma in one, and unknown in one case. All except our case had obstructive hydrocephalus. All cases underwent shunting before developing intracranial pneumocephalus. Pneumocephalus frequently developed in the temporal lobe through bone defect of petrous bone and most of them accompanied intraventricular pneumocephalus. 
A

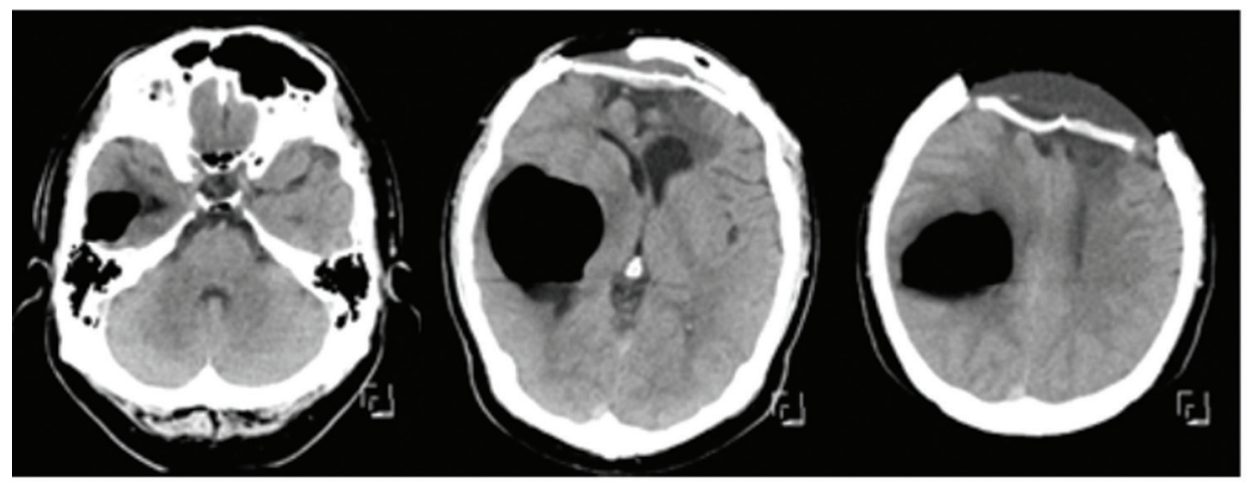

B

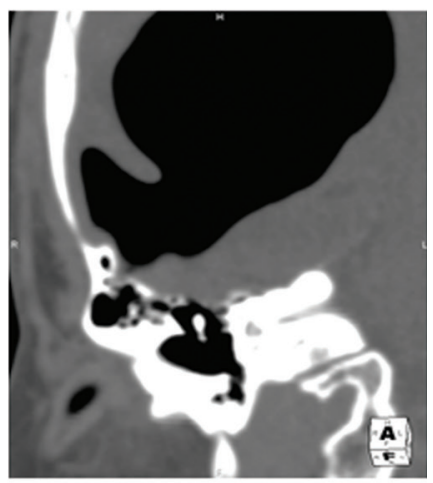

C
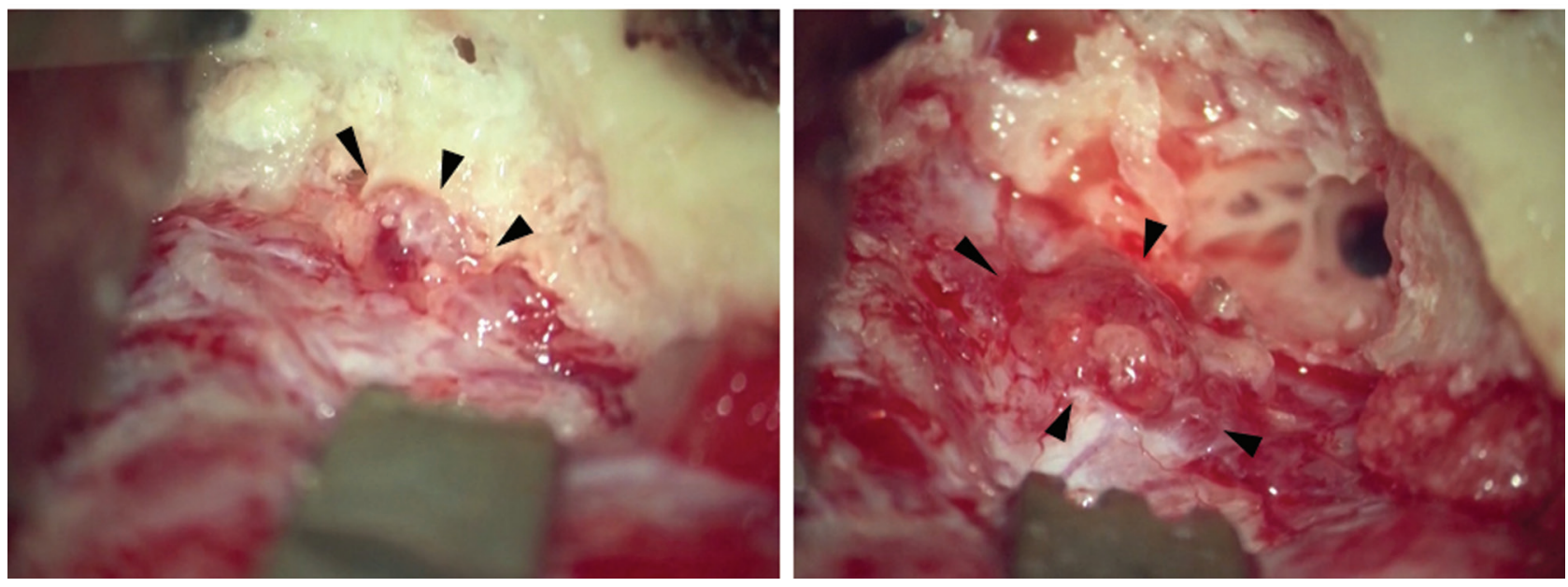

Fig. 2 (A) Axial CT scans demonstrating pneumocephalus in the right temporal lobe. (B) Coronal CT scan demonstrating that intracerebral pneumocephalus continued into the mastoid air cells through a bone defect of the petrous bone. (C) Operative photographs demonstrating the bone defect and encephalocele penetrating into the petrous bone (arrowheads in the left panel), and exposed mastoid air cells and encephalocele after drilling the roof of the mastoid air cells (arrowheads in the right panel). CT: computed tomography.

The present case of intracerebral pneumocephalus demonstrates the cause was preexisting encephalocele in the petrous bone and pressure imbalance caused by LP shunting. This report first demonstrated the preexisting encephalocele prior to the development of intracerebral pneumocephalus.

This finding is important for understanding the pathogenesis of spontaneous pneumocephalus. Communication through a bone defect between the intracranial and extracranial spaces, disruption of the dura mater, and adhesion of the cerebral parenchyma to extracranial space are necessary for the development of intracranial pneumocephalus. ${ }^{1,16)}$ Based on the findings that spontaneous pneumocephalus has developed in patients with chronic elevated ICP secondary to large benign tumor, ${ }^{2)}$ hydrocephalus caused by aqueduct stenosis, ${ }^{11,17)}$ and infratentorial tumor, ${ }^{2,4,8,15)}$ the hypothesis for the mechanism of the development of intracerebral pneumocephalus has been proposed; chronic elevated ICP may be necessary to form the anatomical fistula between the intracranial and extracranial spaces by causing skull base thinning, ${ }^{10)}$ which enlarges a preexisting bone defect or causes a de novo bone defect. Subsequently, the elevated ICP displaces the dura mater and cerebral parenchyma into the bone defect, and forms encephalocele. This hypothesis is partially supported by the evidence of cases in which multiple defects of bone and dura mater were found intraoperatively in the patients with intracerebral pneumocephalus secondary to supratentorial meningioma or hydrocephalus caused by cerebellar astrocytoma or cavernous hemangioma. ${ }^{2,8)}$ However, there have been no reports demonstrating the preexisting encephaloceles or the defects of dura mater so far. The finding of preexisting encephaloceles in this report could provide the evidence of the hypothesis that was speculated in the previous reports. 
A

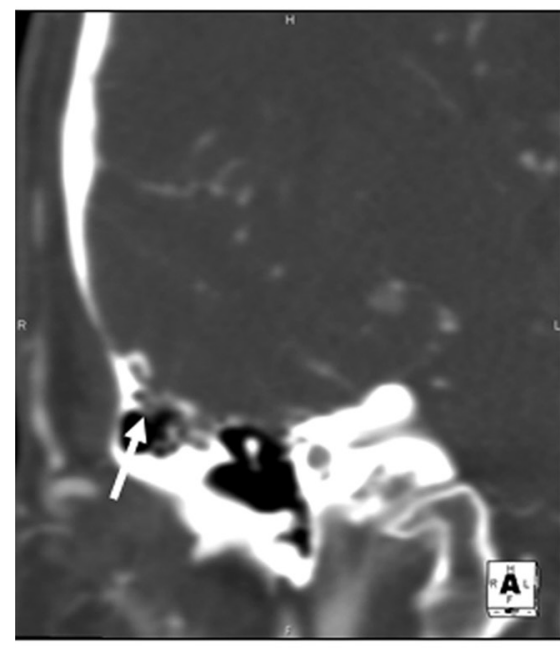

C

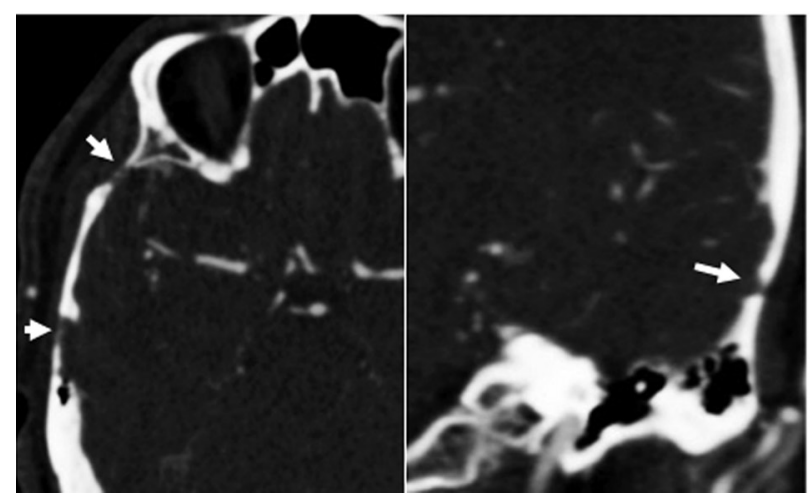

B

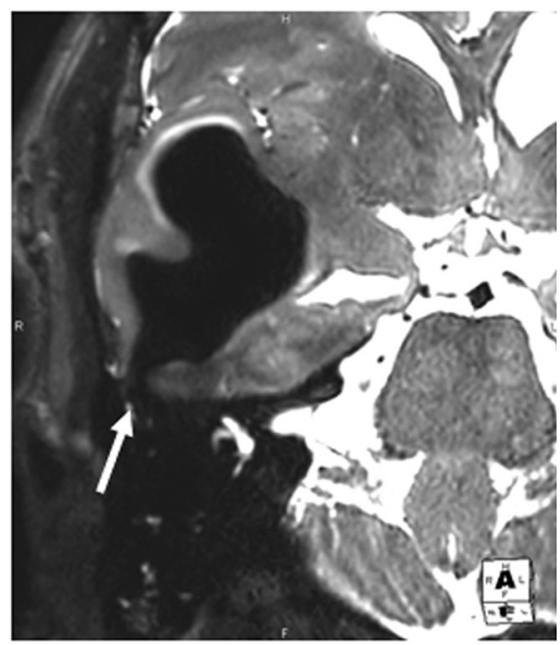

D

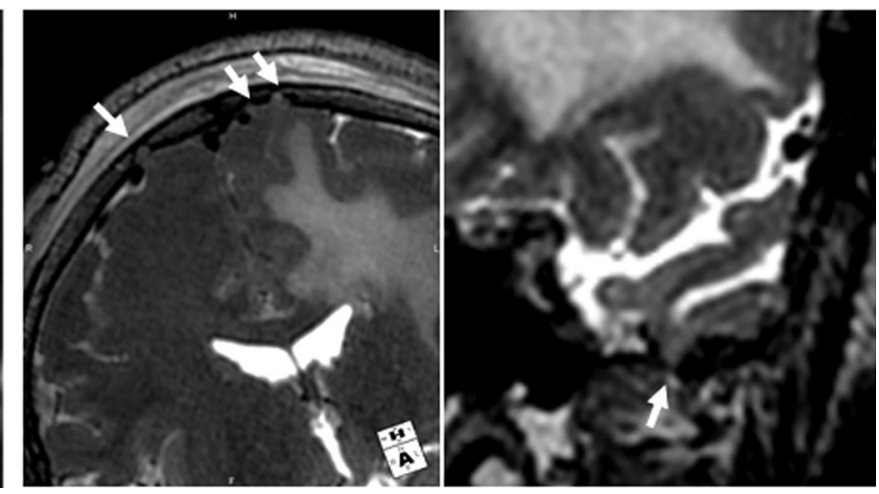

Fig. 3 (A) Coronal CT scan before resection of meningioma demonstrating the preexisting bone defect in the roof of the petrous bone (arrow in the left panel), and coronal 3D CUBE T2-weighted MR image 2 days after resection of meningioma showing encephalocele penetrating into the mastoid air cells (arrow in the right panel). (B) Coronal 3D CUBE T2-weighted MR image at the onset of tension pneumocephalus in the right temporal lobe demonstrating that pneumocephalus was caused by the encephalocele shown in the right panel of Fig. 3A (C) Axial CT scans before resection of meningioma demonstrating multiple bone defects (arrows) in the bilateral temporal bone. (D) Coronal 3D CUBE T2-weighted MR images 2 days after resection of the meningioma demonstrating encephalocele (arrows) in the bilateral calvaria (left panel) and left petrous bone (right panel). CT: computed tomography, MR: magnetic resonance.

The findings in this report can provide the clues to avoid the rare, but serious complication of shunting, especially in the cases with chronic elevated ICP. Preoperatively, the presence of preexisting encephaloceles on heavily T2-weighted MR images or bone defects on thin slice bone CT is useful finding for predicting the development of pneumocephalus after shunting. For such cases, it is recommended to use shunt systems with an anti-siphon device or programmable valve, which was set at high pressure. ${ }^{8,9)}$ After shunting, short- and long-term follow-up is necessary because pneumocephalus could occur from 9 days to 1 year after shunting (Table 1).

The present case report has various limitations. No radiological images were obtained before the onset of meningioma, so no direct evidence was available for the hypothesis that chronic elevated ICP lead to the development of encephalocele in the mastoid air cells. In addition, previous cases with idiopathic normal pressure hydrocephalus also developed pneumocephalus after VP shunting, ${ }^{3)}$ so another unknown mechanism may account for the pathogenesis of spontaneous pneumocephalus.

\section{Conclusions}

The present case demonstrates that pneumocephalus may be caused by the preexisting encephalocele and insertion of LP shunt. Although intracerebral pneumocephalus is a rare complication of VP or LP shunting, 
Table 1 Summary of cases of pneumocephalus due to intracranial tumor and shunting

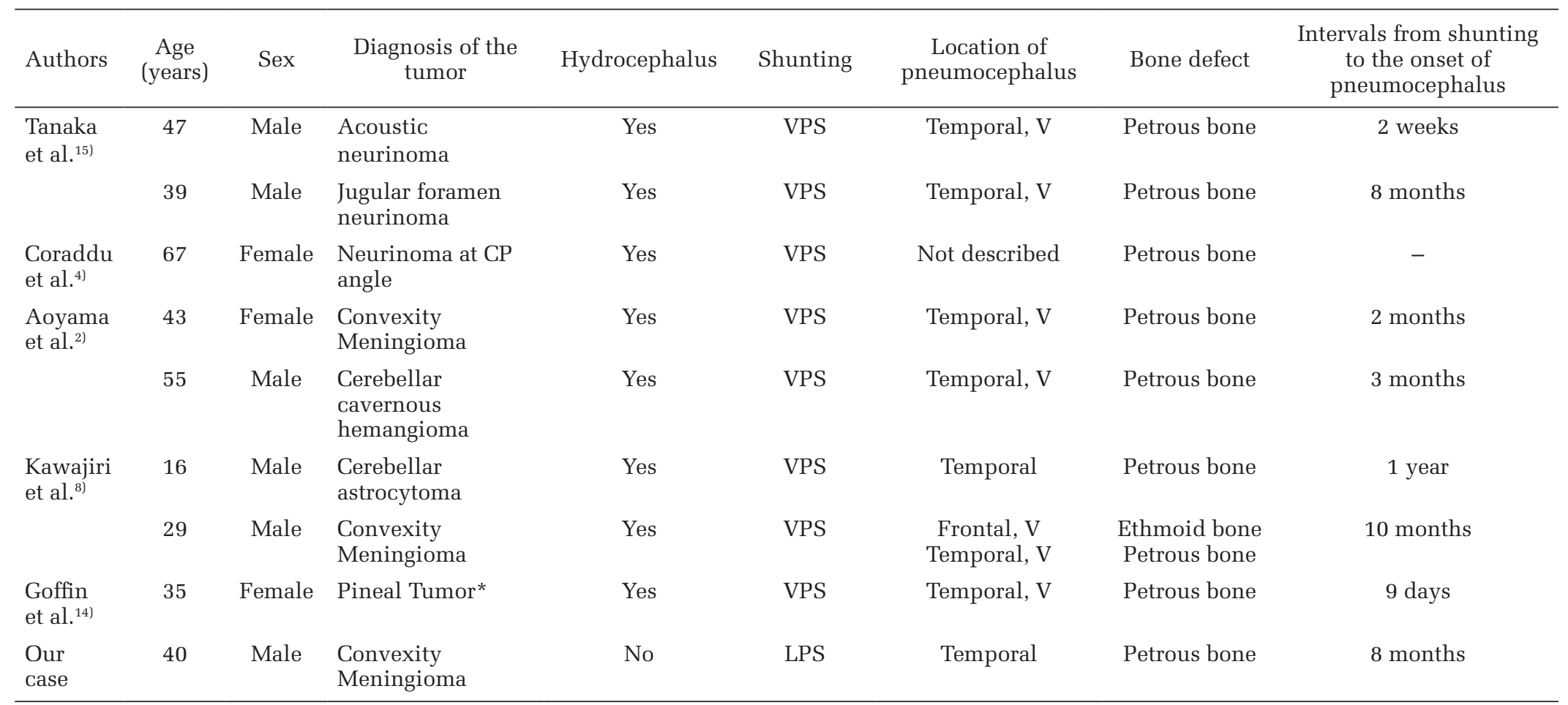

*Unverified histology. CP angle: cerebellopontine angle, LPS: lumbo-peritoneal shunt, V: intraventricular pneumocephalus, VPS: ventriculo-peritoneal shunt. 
the present findings suggest that this complication may be predictable and close follow-up for such cases is indispensable to avoid the severe complication.

\section{Conflicts of Interest Disclosure}

The authors declare that they have no financial conflicts of interest. D.O., T.M., S.N., M.K., and T.T. have registered online Self-reported COI Disclosure Statement Forms through the website for JNS members.

\section{References}

1) Markham JW: The clinical features of pneumocephalus based upon a survey of 284 cases with report of 11 additional cases. Acta Neurochir (Wien) 16: 1-78, 1967

2) Aoyama I, Kondo A, Nin K, Shimotake K: Pneumocephalus associated with benign brain tumor: report of two cases. Surg Neurol 36: 32-36, 1991

3) Barada W, Najjar M, Beydoun A: Early onset tension pneumocephalus following ventriculoperitoneal shunt insertion for normal pressure hydrocephalus: a case report. Clin Neurol Neurosurg 111: 300-302, 2009

4) Coraddu M, Nurchi GC, Floris F, Meleddu V, Uselli S: Intracerebral pneumocephalus after ventriculoperitoneal shunt. Acta Neurol (Napoli) 11: 42-45, 1989

5) Ferrante E, Trimboli M, Cervellino A, Peluso D, Paciello N: Pneumocephalus associated with spontaneous intracranial hypotension. Headache 59: 1093-1094, 2019

6) Hage P, Daou B, Jabbour P: Spontaneous otogenic pneumocephalus due to altitude changes: a case report and review of literature. Clin Neurol Neurosurg 138: 162-164, 2015

7) Honeybul S, Bala A: Delayed pneumocephalus following shunting for hydrocephalus. J Clin Neurosci 13: 939-942, 2006

8) Kawajiri K, Matsuoka Y, Hayazaki K: Brain tumors complicated by pneumocephalus following cerebrospinal fluid shunting--two case reports. Neurol Med Chir (Tokyo) 34: 10-14, 1994

9) Kuba H, Matsukado K, Inamura T, Morioka T, Sasaki M, Fukui M: Pneumocephalus associated with aqueductal stenosis: three-dimensional computed tomographic demonstration of skull-base defects. Childs Nerv Syst 16: 1-3, 2000

10) Rabbani CC, Patel JM, Nag A, et al.: Association of Intracranial Hypertension With Calvarial and Skull Base Thinning. Otol Neurotol 40: e619-e626, 2019

11) Little JR, MacCarty CS: Tension pneumocephalus after insertion of ventriculoperitoneal shunt for aqueductal stenosis. J Neurosurg 44: 383-385, 1976

12) Pitts LH, Wilson CB, Dedo HH, Weyand R: Pneumocephalus following ventriculoperitoneal shunt. Case report. J Neurosurg 43: 631-633, 1975

13) Wannemuehler TJ, Hubbell RD, Nelson RF: Tension pneumocephalus related to spontaneous skull base dehiscence in a patient on BiPAP. Otol Neurotol 37: e322-e324, 2016

14) Goffin J, Plets C: Tension pneumocephalus in association with ventriculoperitoneal shunt. Acta Neurochir (Wien) 76: 121-124, 1985

15) Tanaka A, Matsumoto N, Fukushima T, Tomonaga M: Spontaneous intracerebral pneumocephalus after ventriculoperitoneal shunting in patients with posterior fossa tumors: report of two cases. Neurosurgery 18: 499-501, 1986

16) Jelsma F, Moore DF: Cranial aerocele. Am J Surg 87: 437-451, 1954

17) Ruge JR, Cerullo LJ, McLone DG: Pneumocephalus in patients with CSF shunts. J Neurosurg 63: 532-536, 1985

Corresponding author: Masayuki Kanamori, MD, PhD Department of Neurosurgery, Tohoku University Graduate School of Medicine, 1-1 Seiryomachi, Aoba-ku, Sendai, Miyagi 980-8574, Japan.

e-mail:mkanamori@med.tohoku.ac.jp 